\title{
Nanoparticle Characterization in Pharmaceutical Applications Using Electron Microscopy
}

\author{
K. L. Bunker, J. L. Sturgeon, T. L. Lersch, J. Wolfe, and K. L. Sutch
}

R J Lee Group, Inc., Monroeville, PA 15146

In the pharmaceutical industry, applications of nanotechnology such as diagnostics, drug delivery systems, devices, cosmetics, clothing, food, and supplements are being researched. The Woodrow Wilson International Center of Scholars estimates that there are more than 1,000 manufacturer-identified nanotechnology-based consumer products currently on the market, with over one-third of the products being incorporated into cosmetics, clothing, and sunscreens [1]. These products utilize a variety of nanomaterials, including silver, titanium dioxide, zinc oxide, copper, and alumina nanoparticles.

Drug-delivery systems based on nanotechnology are also of interest in the pharmaceutical industry due to the potential effects on rate of absorption, distribution, and metabolism. Several nanoenabled drug delivery systems either in the research stage or on the market utilize gold nanoparticles, gold nanorods, polymer nanoparticles, liposome nanoparticles, and micelles in addition to a variety of other nanomaterials [2].

Challenges in implementing nanoparticles into different products and drug delivery systems include an understanding of particle size, particle size distribution, particle shape and morphology, elemental composition, as well as agglomeration state. Advances in analytical instrumentation, such as electron microscopy, provide opportunities to obtain information on the nanoparticles and nanomaterials being used in systems and products.

This work focuses on the use of high-resolution scanning electron microscopy (SEM) and scanning transmission electron microscopy (STEM) to identify and characterize a variety of nanoparticles used in the pharmaceutical field. Some of the nanoparticles that will be highlighted are gold nanoparticles, gold nanorods, titanium dioxide, and micelles (Figures 1-3). Different imaging modes such as secondary electron, backscattered electron, bright field-STEM, and dark field-STEM imaging will be shown. An energy dispersive X-ray spectroscopy (EDS) system was also used to acquire elemental information, including spectra and high-resolution X-ray maps (Figure 3). In addition, some of the challenges associated with the electron microscopy analysis of nanoparticles used in the life sciences filed will be discussed, including sample preparation, cryogenic electron microscopy and in-situ electron microscopy.

\section{References:}

[1] Woodrow Wilson International Center of Scholars (www.nanotechproject.org/inventories/consumer).

[2] The Nanotech Revolution in Drug Delivery: Establishing a New Paradigm in Pharmaceuticals, Cientifica Ltd., 2007. 

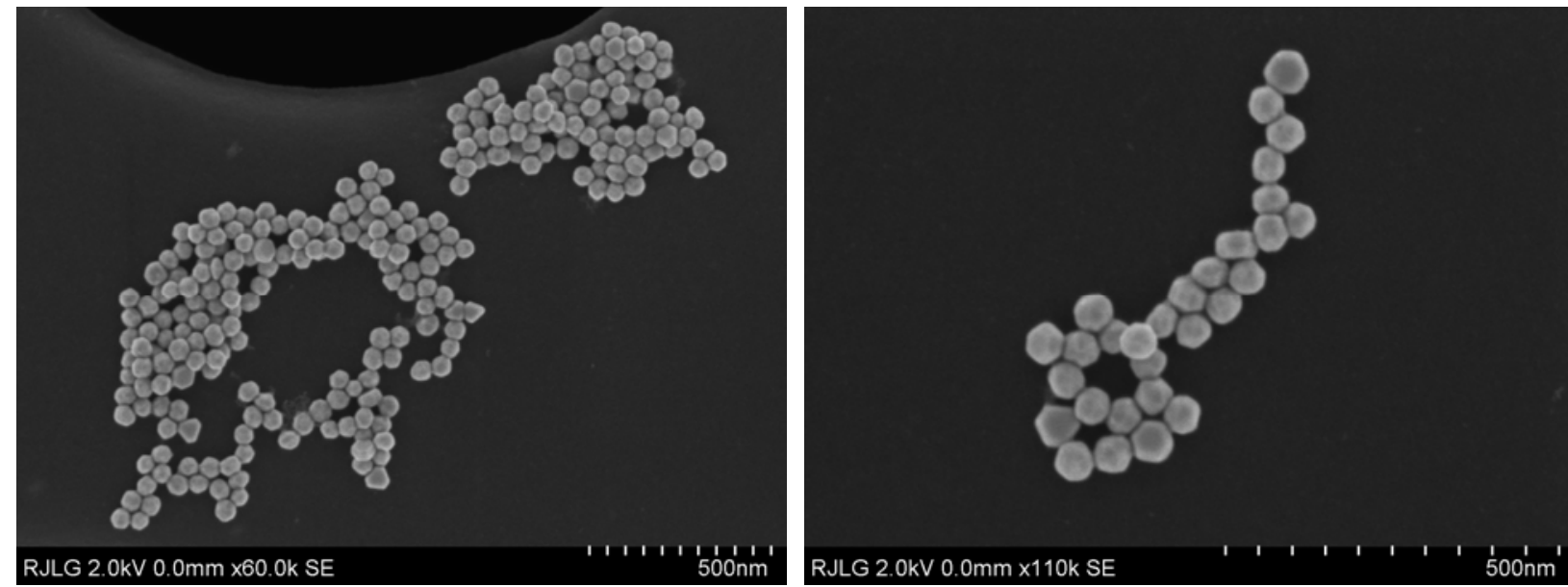

Fig. 1: Secondary electron images of gold nanoparticles, showing a (a) 60kX image and a (b) 110kX image of the gold nanoparticles agglomerated on a holey TEM grid.
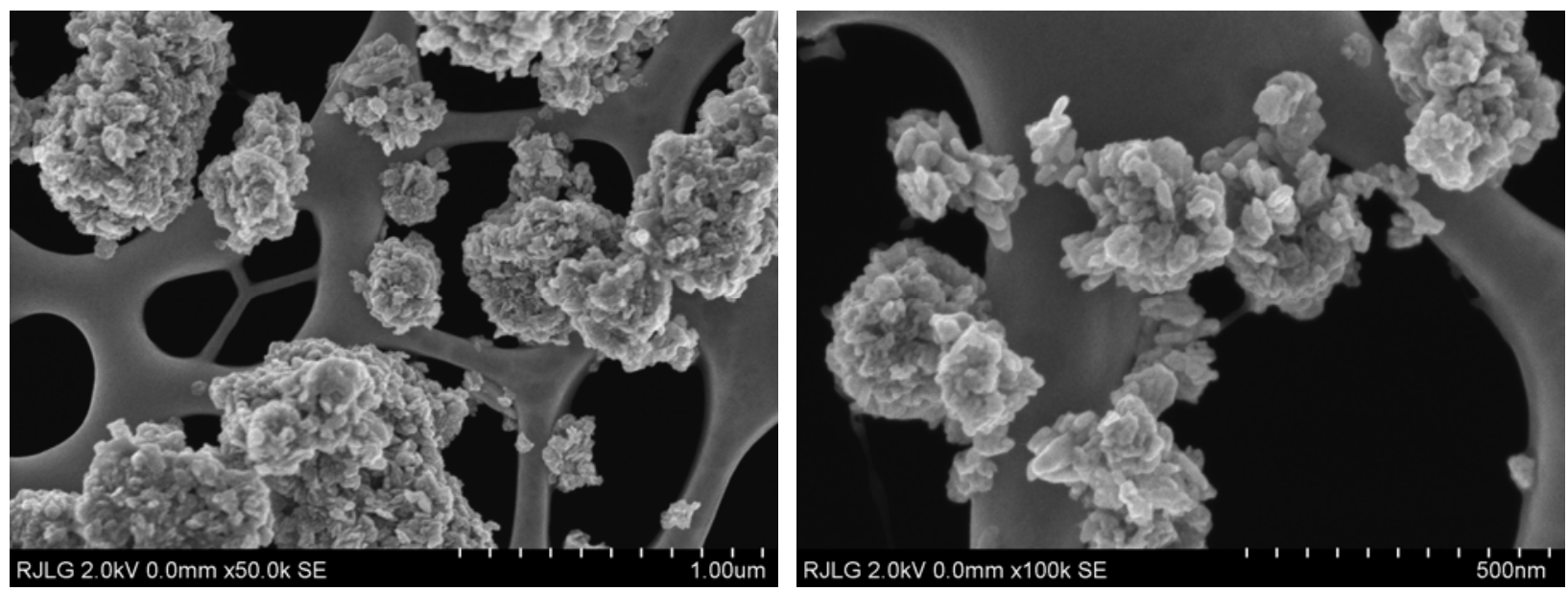

Fig. 2: Secondary electron images of $5 \mathrm{~nm}$ titanium dioxide nanoparticles, showing a (a) $50 \mathrm{kX}$ image and (b) a $100 \mathrm{kX}$ image of the titanium dioxide nanoparticles agglomerated on a lacey TEM grid.
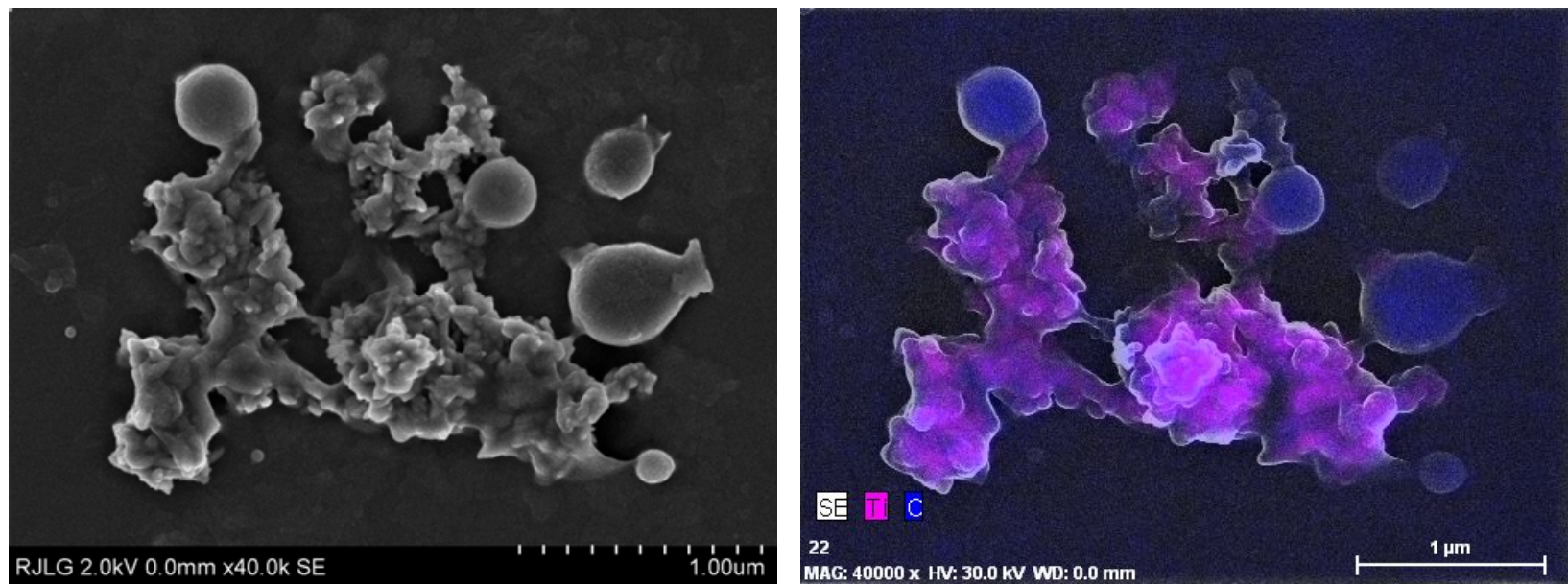

Fig. 3: (a) Secondary electron image of the agglomerated nanoparticles found in sunscreen and (b) an energy dispersive X-ray spectroscopy map showing the location of the titanium dioxide and carbon spheres. 